\title{
Group A Streptococcus detection: Office versus reference laboratory
}

\author{
NICK BURDASH, PhD \\ JOHN J. FERNANDES, DO
}

Clinical differentiation between a virus and $\beta$-hemolytic streptococci as a cause of pharyngitis remains difficult. In the physician's laboratory, more rapid procedures using immunologic methods have replaced culture methods, traditionally requiring 2 to 3 days for identification of $\beta$-hemolytic streptococci. Attention to the diagnostic sensitivity and specificity of these procedures will guide appropriate use.

(Key words: Laboratory procedures, diagnosis, group A Streptococcus, pharyngitis)

The advancement of medical science, rather than simplifying, has made the problems surrounding treatment decisions regarding pharyngitis, especially in young patients, more complex.

Originally, the differential diagnostic dilemma was based on the fact that the most likely causative agents of sore throat-barring unusual circumstances-were any of several viruses or Lancefield group A $\beta$-hemolytic strep-

From the Department of Pathology and Laboratory Medicine, Philadelphia College of Ostoepathic Medicine, Philadelphia, $\mathrm{Pa}$.

Currently, Dr Burdash is professor of immunology and microbiology and director of research, Department of Anesthesiology, College of Medicine, University of South Florida, Tampa, and Dr Fernandes is professor of pathology, Department of Pathology, Olympia Fields Osteopathic Hospital and Medical Center, Olympia Fields, IL 60461 .

Reprint requests to Nick Burdash, $\mathrm{PhD}$, Department of Anesthesiology, College of Medicine, University of South Florida, MDC59, Tampa, FL 33612. tococci (BHS) (Streptococcus pyogenes). Most often, streptococcal infection is self-limiting; however, such infection can spread, invading other tissues and causing more serious disease. The risk of rheumatic fever and poststreptococcal glomerulonephritis is a primary reason for the treatment of the original infection.

Rheumatic fever, which had been slowly disappearing in the United States, has made a significant comeback over the past few years. Although the relationship between group A BHS pharyngitis and rheumatic fever has been known and studied for many years, the specific cause remains obscure. Because the viruses that cause pharyngitis are essentially nonsusceptible to antimicrobial agents, and because these disease are self-limiting and rarely initiate other maladies, the two agents must be clearly differentiated as accurately and rapidly as possible.

\section{Traditional microbiologic methods}

The clinical diagnosis of these two specific disease is highly inaccurate and cannot be relied on, even among the most experienced practitioners, and no indirect laboratory indicators have been discovered that are accurate enough for differentiation. For many years, detection of pharyngeal group A BHS was therefore limited to traditional microbiologic methods involving significant amounts of time. Unfortunately, in most clinical microbiology laboratories, these same methods have not changed and are still in use today.

Processing of throat swabs in the laboratory involves specimen inoculation onto blood agar 


\begin{tabular}{|c|c|c|c|c|c|c|c|c|}
\hline \multirow[b]{4}{*}{ Antigen detection test } & \multicolumn{8}{|c|}{$\begin{array}{l}\text { Table } \\
\text { Highest Dilution (Minimum Concentration) Among Four Strains of Group A } \\
\text { Streptococci in Broth Required to Produce a Positive Antigen Test Result }\end{array}$} \\
\hline & \multicolumn{4}{|c|}{$\begin{array}{l}\text { Highest dilution } \\
\text { yielding a positive } \\
\text { test result* }\end{array}$} & \multicolumn{4}{|c|}{$\begin{array}{l}\text { Minimum detectable No. of } \\
\text { colony-forming units }\end{array}$} \\
\hline & \multicolumn{4}{|c|}{ Strains } & \multicolumn{4}{|c|}{ Strains } \\
\hline & 1 & 2 & 3 & 4 & 1 & 2 & 3 & 4 \\
\hline Ventrescreen (EIA) & 2 & 2 & 2 & 1 & $6.3 \times 10^{4}$ & $7.2 \times 10^{4}$ & $6.9 \times 10^{4}$ & $3.2 \times 10^{5}$ \\
\hline Directigen (LA) & 3 & 2 & 2 & 1 & $6.3 \times 10^{3}$ & $7.2 \times 10^{4}$ & $6.9 \times 10^{4}$ & $3.2 \times 10^{5}$ \\
\hline Abbott (EIA) & 3 & 3 & 2 & 1 & $6.3 \times 10^{3}$ & $7.2 \times 10^{3}$ & $6.9 \times 10^{4}$ & $3.2 \times 10^{5}$ \\
\hline Reveal (LA) & 2 & 2 & 2 & 1 & $6.3 \times 10^{4}$ & $7.2 \times 10^{4}$ & $6.9 \times 10^{4}$ & $3.2 \times 10^{5}$ \\
\hline Culturette (LA & 3 & 2 & 2 & 1 & $6.3 \times 10^{3}$ & $7.2 \times 10^{4}$ & $6.9 \times 10^{4}$ & $3.2 \times 10^{5}$ \\
\hline Cards (EIA) & 2 & 2 & 2 & 1 & $6.3 \times 10^{4}$ & $7.2 \times 10^{4}$ & $6.9 \times 10^{4}$ & $3.2 \times 10^{5}$ \\
\hline Icon Access (EIA) & 4 & 4 & 4 & 2 & $6.3 \times 10^{2}$ & $7.2 \times 10^{2}$ & $6.9 \times 10^{2}$ & $3.2 \times 10^{4}$ \\
\hline Icon Tandem (EIA) & 4 & 3 & 3 & 3 & $6.3 \times 10^{2}$ & $7.2 \times 10^{3}$ & $6.9 \times 10^{3}$ & $3.2 \times 10^{3}$ \\
\hline \multicolumn{9}{|c|}{$\begin{array}{l}\text { *Logarithm of dilution factor. } \\
\text { EIA = enzyme immunoassay; LA = latex agglutination. } \\
\text { Reprinted by permission from White CB, Lieberman MM, Morales E: An in vitro comparison of eight rapid streptococcal antigen detection tests. } \\
\text { Pediatr 1988; } 113: 691-693 .\end{array}$} \\
\hline
\end{tabular}

plates followed by incubation over night. The next day, colonies that show $\beta$ hemolysis are inoculated onto a second blood agar plate along with a filter paper disc impregnated with bacitracin. In general, group A BHS are susceptible to bacitracin, whereas other Lancefield groups are not. Plates are observed for bacitracin inhibition after a second overnight incubation.

Microbiologists are in general agreement that bacitracin susceptibility testing is too inaccurate when performed on the primary plate and should be performed on the pure culture BHS or the second plate. Therefore, depending on the time a specimen is submitted to the laboratory and processing is initiated, the final reporting of results will take minimally 2 days, and frequently can stretch to 3 days.

\section{Immunologic testing}

Some laboratories may elect to identify group A BHS from the isolated $\beta$-hemolytic colonies on the primary blood agar plate by using immunologic testing. In this scenario, medium inoculated with the throat specimen still undergoes overnight incubation with suspected $\beta$-hemolytic colonies tested for the group A antigen the next day. This method almost always provides results in 1 day. The difference in methods is directly related to the increased cost of performing an immunologic assay, as opposed to the inexpensive bacitracin disc. Many laboratories also perform direct testing for group A BHS from throat swabs and offer this procedure on a "stat" basis. Although the test takes only minutes to perform, patients often have to wait for hours because of the inherent paper work, "getting to the specimen," and then the reporting of results.

The availablity of immunologic identification kits for direct, rapid detection of group A BHS has also caused certain complications in the physician's office laboratory. Dozens of brand name kits are available for identification of Lancefield streptococcal groups. Not all are recommended for direct testing from patient samples, and many require several steps to extract the Lancefield group antigenic portion of the organism before the antigen-antibody reaction can take place.

Currently, two main detection methods are available, both dependent on specific antibody to the group A antigen. The older, simpler method involves the agglutination of small particles coated with antibody to group A antigen. Agglutination indicates a positive reaction, whereas nonagglutination indicates no streptococcal antigens present. 
The difference between a positive and negative test is not as simple to discern as one might suspect. Controls, adhering to instructions, and a trained, experienced staff ensure more accurate results. The other more recently developed methods involve labeling the antibody with an enzyme. If a positive reaction occurs, the addition of substrate will result in a color change readily visible to the naked eye. These solid-phase enzyme immunoassays are generally more expensive and range from two steps to more than half a dozen. Newer methods such as nucleic acid probes are commercially available for other organisms, and surely will be available for BHS as methods are improved.

At present, the major problem of direct detection of any organism resulting in detectable antigen-antibody complexes involves the classic sensitivity-specificity dilemma. As the ability to detect all true streptococcal infections (sensitivity) increases, the ability to determine that no streptococcal infection exists (specificity) decreases. As an assay increases in sensitivity, it loses its ability to distinguish between low levels of streptococcal antigen and other closely related antigens and/or other background factors become evident causing nonantigenic agglutinations or color change in a test, resulting in increased proportions of falsepositive reactions.

If an assay is too specific, it may be unable to detect low levels of streptococcal antigen, thereby providing false-negative tests and resulting in nontreatment of true streptococcal pharyngitis, a much more serious situation. In practice, it appears from the literature that most direct tests are less than $95 \%$ sensitive and many are far less than $90 \%$ sensitive. ${ }^{1,2}$ The vast majority of kits have little chance ( $1 \%$ or less) of false-negative determination.

Many of those practitioners who choose to perform direct testing for group A BHS in an outpatient setting also send a duplicate specimen to the microbiology laboratory. ${ }^{3}$ In this way, if the direct test is negative, a backup routine culture result should be available in 24 to 48 hours.

There seems to be little consensus as to a brand or method preferred among kit users. ${ }^{4}$
There are dozens of articles and published abstracts comparing an individual detection kit with culture. However, the variables of these studies preclude comparison of kits. Examples of differences in the individual studies include use of single or double swabs, aerobic or anaerobic culture incubation, routine or special culture media, number of specimens, number of positive specimens, and performance by certified laboratory personnel vs office personnel.

For a more comprehensive view of comparisons, the reader is referred to an article by White and colleagues, ${ }^{5}$ who tested eight antigen detection kits using varying dilutions of different laboratory group A BHS strains (Table). Unfortunately, as kits come on and off the market and other kits are "improved," the choices and variables are never ending. A comparison of direct testing with culture is often necessary to determine what works best in a given situation. A short conference with a clinical microbiologist often leads to a concise plan to evaluate one or more identification systems.

Availability of a more foolproof method for discriminating between viral and streptococcal pharyngitis, attention to detail in obtaining the proper specimen, ensuring its rapid transport to the laboratory, or carrying out direct identification in the office laboratory through use of proper technique and quality control will substantially increase the odds of accurate detection and identification. ${ }^{6}$

\section{References}

1. Burdick JR, Sottile W: Comparison of five latex agglutination kits for detecting group A streptococcal antigen. JAOA 1988;88:219-224.

2. Roddey OF, Clegg HW, Clardy LT, et al: Comparison of a latex agglutination test and four culture methods for identification of group A streptococcus in a pediatric office laboratory. $J$ Pediatr 1986;108:347-351.

3. White CB, Harris R, Weir MR, et al: Streptococcal pharyngitis: Comparison of latex agglutination and throat culture. Clin Pediatr 1988;27:431-435.

4. Radetsky M, Solomon JA, Todd JK: Identification of streptococcal pharyngitis in the office laboratory: Reassessment of new technology. Pediatr Infect Dis J 1987;6:556-563.

5. White CB, Lieberman MM, Morales E: An in-vitro comparison of eight rapid streptococcal antigen detection tests. $J P e$ diatr 1988;113:691-693.

6. Willis SE: Throat culture or rapid strep test. Postgrad Med 1990;88:111-114. 